\title{
Synthesis of heparosan oligosaccharides by Pasteurella multocida PmHS2 single-action transferases
}

\author{
Anaïs A. E. Chavaroche • \\ Lambertus A. M. van den Broek • Carmen Boeriu • \\ Gerrit Eggink
}

Received: 12 October 2011 / Revised: 29 November 2011 / Accepted: 1 December 2011 /Published online: 24 December 2011

(C) The Author(s) 2011. This article is published with open access at Springerlink.com

\begin{abstract}
Pasteurella multocida heparosan synthase PmHS2 is a dual action glycosyltransferase that catalyzes the polymerization of heparosan polymers in a nonprocessive manner. The two PmHS2 single-action transferases, obtained previously by site-directed mutagenesis, have been immobilized on $\mathrm{Ni}(\mathrm{II})$-nitrilotriacetic acid agarose during the purification step. A detailed study of the polymerization process in the presence of non-equal amounts of PmHS2 single-action transferases revealed that the glucuronyl transferase (PmHS2-GlcUA ${ }^{+}$) is the limiting catalyst in the polymerization process. Using experimental design, it was determined that the $N$-acetylglucosaminyl transferase (PmHS2-GlcNAc ${ }^{+}$) plays an important role in the control of heparosan chain elongation depending on the number of heparosan chains and the UDP-sugar concentrations present in the reaction mixture. Furthermore, for the first time, the synthesis of heparosan oligosaccharides alternately using PmHS2-GlcUA ${ }^{+}$and PmHS2-GlcNAc ${ }^{+}$is reported. It was shown that the synthesis of heparosan oligosaccharides by PmHS2 single-action transferases do not require the presence of template molecules in the reaction mixture.
\end{abstract}

Electronic supplementary material The online version of this article (doi:10.1007/s00253-011-3813-2) contains supplementary material, which is available to authorized users.

A. A. E. Chavaroche · G. Eggink

Bioprocess Engineering Group,

Wageningen University and Research Center,

P.O. Box 8129, $6700 \mathrm{EV}$, Wageningen, the Netherlands

A. A. E. Chavaroche $\cdot$ L. A. M. van den Broek $\cdot$ C. Boeriu

G. Eggink $(\square)$

Food and Biobased Research,

Wageningen University and Research Center,

P.O. Box 17, 6700 AA, Wageningen, the Netherlands

e-mail: gerrit.eggink@wur.nl
Keywords Glycosaminoglycan · Heparosan ·

Oligosaccharide $\cdot$ Heparosan synthase $\cdot$ Single-action transferase $\cdot$ Polymerization

\section{Introduction}

Heparin (Hep) and heparan sulfate (HS) are analog molecules belonging to the glycosaminoglycan (GAG) family. $\mathrm{Hep} / \mathrm{HS}$ polymers are ubiquitous in mammalian cells and are involved in many physiological processes (Rabenstein 2002; Linhardt and Toida 2004). Heparin is a well-known pharmaceutical compound that is used in large amounts in surgery for its anticoagulant properties. Heparan sulfate, an analog of heparin, is not used yet for therapeutic purposes, but it is considered to have a large potential for medical applications (Rabenstein 2002; Lindahl 2007). Currently, pharmaceutically grade heparin anticoagulant products are mainly obtained from animal tissues, and some are chemically synthesized. Neither of these systems enable the production of a large range of well-defined Hep/HS oligomers and polymers; therefore, alternative processes to control Hep/HS synthesis are of interest (Laremore et al. 2009).

Heparosan is the unsulfated precursor of heparin and heparan sulfate in mammalians. In the pathogenic bacteria Escherichia coli K5 (Vann et al. 1981), Pasteurella multocida type D (Pandit and Smith 1993; Rimler 1994), and Avibacterium paragallinarum genotype II ( $\mathrm{Wu}$ et al. 2010), heparosan is a component of the polysaccharide capsule. Heparosan can be produced either by extracting it from microorganisms or by using recombinant enzymes in vitro. The use of $E$. coli $\mathrm{K} 5$ to produce heparosan in largescale fermentation has been reported and enabled the recovery of $15 \mathrm{~g} / \mathrm{L}$ of heparosan (Wang et al. 2010). Despite the fact that this production strategy is cost-effective, neither the 
heparosan chain length nor the incorporation of modified and analog sugar residues can be controlled.

Heparosan can also be synthesized in vitro using recombinant heparosan synthases. Heparosan synthases from mammalians (EXT1 and EXT2) (Mccormick et al. 2000; Kim et al. 2003), Drosophila (TTV, SOTV, and BOTV) (Bellaiche et al. 1998; Izumikawa et al. 2006), E. coli K5 (KfiA and KfiC) (Griffiths et al. 1998; Hodson et al. 2000; Sugiura et al. 2010), and P. multocida type D (PmHS1 or PmHS2) (Sismey-Ragatz et al. 2007; Chavaroche et al. 2010) have been characterized. EXT1 is a dual action transferase, while EXT2 and $\mathrm{Kfi}$ ( $\mathrm{KfiA}$ and $\mathrm{KfiC}$ ) heparosan synthases are mono-action transferases that catalyze only one transferase activity; thus, both transferases are necessary (EXT1 and EXT2 or KfiA and $\mathrm{KfiC}$ ) for polymerization activity (Busse and Kusche-Gullberg 2003; Sugiura et al. 2010). In contrast, PmHS heparosan synthases (PmHS1 and PmHS2) are both dual action glycosyltransferases exhibiting a glucuronyl transferase and an $N$-acetylglucosaminyl transferase activity. PmHS single-action transferase enzymes (PmHS-GlcUA ${ }^{+}$and PmHS-GlcNAc ${ }^{+}$) were obtained by applying site-directed mutagenesis on the DXD motif of PmHS1 (Kane et al. 2006) or PmHS2 (Chavaroche et al. 2011). The PmHS2-GlcUA ${ }^{+}$and PmHS2-GlcNAc ${ }^{+}$incubated together polymerized heparosan chains (Chavaroche et al. 2011).

There is a close relationship between the Hep/HS structures and their biological activity (Bishop et al. 2007). Indeed, it was reported that heparin pentasaccharides require the presence of iduronic acid to exhibit anticoagulant activity, while decasaccharide heparin exhibit anticoagulant activity in the absence of iduronic acid (Chen et al. 2007). Moreover, heparan sulfate proteoglycans are involved in many aspects of cancer development from cell growth to metastasis. In this pathology, the chain length of the polymers influences the biological activity. High molecular weight heparin enhances the binding of the growth factors to their receptors and thus promotes angiogenesis, while low molecular weight heparin has the opposite effect by inhibiting their binding (Yip et al. 2006; Lazo-Langner et al. 2007). The relation between structure and biological activity is also true for the antiviral activity of Hep/HS, which can also be modulated by varying the degree of epimerization, sulfation, or/and the chain length (Chen et al. 1997; Rusnati et al. 2009). Thus, in order to synthesize heparin and heparan sulfate with specific chain length, it is important to control the elongation of the polymer backbone heparosan. Recently, the controlled synthesis of heparosan-like oligosaccharides has been reported by applying alternately the activity of the glycosyltransferases KfiA ( $N$-acetyl-D-glucosaminyl transferase) and PmHS2 ( $N$-acetylglucosaminyl transferase and glucuronyl transferase) in the presence of UDP-sugars and a disaccharide acceptor (GlcUA-AnMannose) (Liu et al. 2010;
$\mathrm{Xu}$ et al. 2011). Nevertheless, due to the dual action of PmHS2, the synthesis had to be carefully monitored. The controlled synthesis of glycosaminoglycan hyaluronan and chondroitin oligosaccharides has also been reported. Immobilized P. multocida hyaluronan synthase PmHAS single-action transferases (Deangelis et al. 2003) and immobilized E. coli chondroitin polymerase K4CP single-action polymerases (Sugiura et al. 2008) synthesized monodisperse 20-mer hyaluronan chains and 16-mer chondroitin chains from short oligosaccharide templates, respectively.

Here we report the use of an immobilized-metal affinity chromatography (IMAC) technique for the immobilization of PmHS2 single-action transferases (PmHS2-GlcNAc ${ }^{+}$and PmHS2-GlcUA $^{+}$). PmHS2 enzymes immobilized with $\mathrm{Ni}$ (II)-nitrilotriacetic acid (Ni-NTA) agarose were characterized for their polymerization activity, stability, and ability to elongate step-by-step heparosan oligosaccharides. We observed that the polymerization process is influenced by both transferase activities and both UDP-sugar concentrations, which make the control of heparosan chain elongation more complicated. However, we were able to show for the first time that both PmHS2 single-action transferases can be used in an alternate manner and in the absence of oligosaccharide templates to synthesize heparosan oligosaccharides. This study opens new perspectives for the synthesis of defined length heparosan oligosaccharides.

\section{Experimental procedure}

Protein expression, purification, and immobilization

The genes pmhs2-GlcUA ${ }^{+}$and pmhs2-GlcNAc ${ }^{+}$were ligated into pET101 and transformed in E. coli BL21*(DE3) (Invitrogen) (Chavaroche et al. 2011). The recombinant proteins PmHS2-GlcUA ${ }^{+}$and PmHS2-GlcNAc ${ }^{+}$were expressed with a V5-epitope and a polyhistidine tag (HisTag) at their $\mathrm{C}$ terminus.

The protein expression and the protein recovery procedures were performed as previously described (Chavaroche et al. 2010, 2011). The soluble fraction $(4 \mathrm{~mL})$ obtained from $100 \mathrm{~mL}$ of induced $E$. coli culture was added to $1 \mathrm{~mL}$ of Ni-NTA agarose slurry (50:50\% agarose/buffer) (Qiagen). In order to purify and desalt PmHS2 transferases (PD-10 purified), both the immobilization step on Ni-NTA agarose and the desalting step of the purified fraction using PD-10 desalting column were performed. In order to immobilize PmHS2-GlcUA ${ }^{+}$and PmHS2-GlcNAc ${ }^{+}$on Ni-NTA agarose, the His-Tag purification was slightly modified. The elution step (phosphate buffer, $250 \mathrm{mM}$ of imidazole) was omitted, and the subsequent desalting step (PD10 column) was removed. Instead, the PmHS2 single-action transferases/Ni-NTA agarose mixture was washed and 
equilibrated five times with Tris- $\mathrm{HCl}(50 \mathrm{mM}, \mathrm{pH} 7.15)$, then three times with Tris- $\mathrm{HCl}(50 \mathrm{mM}, \mathrm{pH} 7.15), \mathrm{Mn}^{2+} / \mathrm{Mg}^{2+}$ $(11: 11 \mathrm{mM})$. Prior to being stored at $4{ }^{\circ} \mathrm{C}$ or to be used in polymerization assay, 0.50 to $1.75 \mathrm{~mL}$ of Tris- $\mathrm{HCl}(50 \mathrm{mM}$, $\mathrm{pH} 7.15), \mathrm{Mn}^{2+} / \mathrm{Mg}^{2+}$ (4 mM each) was added to the PmHS2GlcUA $^{+} /$agarose and/or PmHS2-GlcNAc ${ }^{+}$/agarose (referred as immobilized enzymes).

The amount of protein immobilized onto the Ni-NTA resin was determined by a bicinchoninic acid assay (Pierce) using bovine serum albumin as the standard. The efficiency of the protein binding during the washing steps and the storage was evaluated by sodium dodecyl sulfatepolyacrylamide gel electrophoresis (SDS-PAGE) analysis (Chavaroche et al. 2010).

\section{Standard polymerization condition}

The polymerization reaction with a final volume between 30 and $200 \mu \mathrm{L}$ contained $40 \mathrm{mM}$ Tris $-\mathrm{HCl}(\mathrm{pH} 7.15), 4 \mathrm{mM}$ $\mathrm{MnCl}_{2}, 4 \mathrm{mM} \mathrm{MgCl}_{2}$, UDP-GlcUA, UDP-GlcNAc, and PD10-purified or immobilized recombinant PmHS2 singleaction transferases. Unless otherwise mentioned, the enzymes were freshly prepared. The amount of UDP-sugar and enzymes varied with the experiments; details are given in the figure legends. The reactions were performed in the dark at $30-32^{\circ} \mathrm{C}$ under mild shaking conditions ranging from 2 to $24 \mathrm{~h}$.

In order to compare the storage stability of purified and immobilized enzymes, the same amount of, either, PD10purified or immobilized single-action transferases was re-suspended in $3.5 \mathrm{~mL}$ buffer and stored at $4{ }^{\circ} \mathrm{C}$ in the presence of Tris- $\mathrm{HCl}$ (50 mM, pH 7.15), $\mathrm{Mn}^{2+} / \mathrm{Mg}^{2+}$ (4 mM each). At several time points, aliquots of the enzymes were incubated at $30-32^{\circ} \mathrm{C}$ in the presence of UDP-sugars. The polymerization efficiency was compared by monitoring the conversion of UDP-sugars by the coupled enzyme assay.

\section{Experimental design}

To determine the relationship between the ratio of transferases, the UDP-sugar concentrations, and the heparosan size distribution, samples were prepared according to a fractional factorial design of four variables with each three levels $\left(3^{4-1}\right)$. Preliminary experiments were performed to determine the lowest PmHS2 single-action transferase and UDP-sugar concentrations needed to observe activity. From these results, the concentrations for each variable were determined (Table 1). This experimental design consisted in the preparation of 27 reaction mixtures in which different combinations of UDP-sugars and PmHS2 single-action transferase concentrations were added together (Supplementary information Table 1). The samples were incubated for $4 \mathrm{~h}$ to avoid the complete conversion of the UDP-sugars when low UDP-sugar concentrations were present; each reaction mixture was analyzed for UDP content (coupled enzyme assay) and polymer chain length (high-performance size exclusion chromatography, HPSEC).

To analyze the data, it was decided to use the so-called response surface methodology described by Box and Draper (1987). The analysis of the data was done by performing a linear regression in which 15 parameters (main effects, quadratic effects, and interaction effects) were taken into account. The response variable $(y)$ was modeled using the following equation:

$$
\begin{aligned}
y= & b_{0}+b_{1} x_{1}+b_{2} x_{2}+b_{3} x_{3}+b_{4} x_{4}+b_{5} x_{1}^{2}+b_{6} x_{2}{ }^{2} \\
& +b_{7} x_{3}^{2}+b_{8} x_{4}^{2}+b_{9} x_{1} x_{2}+b_{10} x_{1} x_{3}+b_{11} x_{1} x_{4} \\
& +b_{12} x_{2} x_{3}+b_{13} x_{2} x_{4}+b_{14} x_{3} x_{4}
\end{aligned}
$$

In this case, $y$ is the heparosan molecular weight, $b_{0}$ is the intercept, $b_{1}$ to $b_{14}$ the regression coefficients, and $x_{1}, x_{2}, x_{3}$, and $x_{4}$ are the variables: PmHS2-GlcNAc ${ }^{+}$, PmHS2GlcUA $^{+}$, UDP-GlcUA, and UDP-GlcNAc concentration, respectively. The estimation of the regression coefficients was done by least squares regression.

The calculation of the so-called Mallow's Cp criterion was used to determine the best fit for this model. The Mallow's Cp criterion is calculated by taking into account the fit of the model and giving penalties for each additional parameter used to fit a model to the data. Thus, the best

\begin{tabular}{|c|c|c|c|c|c|}
\hline & & \multicolumn{4}{|l|}{ Variables } \\
\hline & & $\begin{array}{l}x_{1} \\
\text { PmHS2-GlcNAc }\end{array}$ & $\begin{array}{l}x_{2} \\
\text { PmHS2-GlcUA }\end{array}$ & $\begin{array}{l}x_{3} \\
\text { UDP-GlcUA }\end{array}$ & $\begin{array}{l}x_{4} \\
\text { UDP-GlcNAc }\end{array}$ \\
\hline \multirow[t]{3}{*}{ Levels } & Low & 7 & 7 & 0.5 & 0.5 \\
\hline & Medium & 21 & 21 & 2.75 & 2.75 \\
\hline & High & 34 & 34 & 5 & 5 \\
\hline
\end{tabular}
model to represent the data is obtained when the Mallow's

Table 1 Variables used for the fractional factorial design of four variables and three levels $\left(3^{4-1}\right)$

The variables $x_{1}$ and $x_{2}$ are the PmHS2 single-action transferase concentrations (micrograms per milliliter), and $x_{3}$ and $x_{4}$ are the UDP-sugar concentrations (millimolars), respectively 
$\mathrm{Cp}$ is at the lowest value. The visualization of the response is given by a contour plot ("Results" section). In practice, the response variable can only be visualized as a function of two independent variables. Therefore, cross sections at different levels of the two other variables were made. The visualization was done using the mathematical software package Matlab.

Step-by-step elongation of heparosan oligosaccharides

Immobilized PmHS2 single-action transferases $(0.80 \pm$ $0.05 \mathrm{mg} / \mathrm{mL}$ Ni-NTA agarose) were transferred separately to two empty disposable PD10 columns (GE Healthcare): PmHS2-GlcUA ${ }^{+}$(column A) and PmHS2-GlcNAc ${ }^{+}$ (column B) (Fig. 1). Elongation of heparosan oligosaccharide was performed by addition of polymerization buffer (Tris- $\mathrm{HCl}$ (50 mM, pH 7.15), $\mathrm{Mn}^{2+} / \mathrm{Mg}^{2+}$ (4 mM each), and UDP-sugars ( 3.25 up to $6.50 \mathrm{mM}$ ) to the columns. With the exception of the initiation step in column A (PmHS2$\mathrm{GlcUA}^{+}$) to which both UDP-sugars were added, each of the following incubation steps contained only UDP-GlcUA or

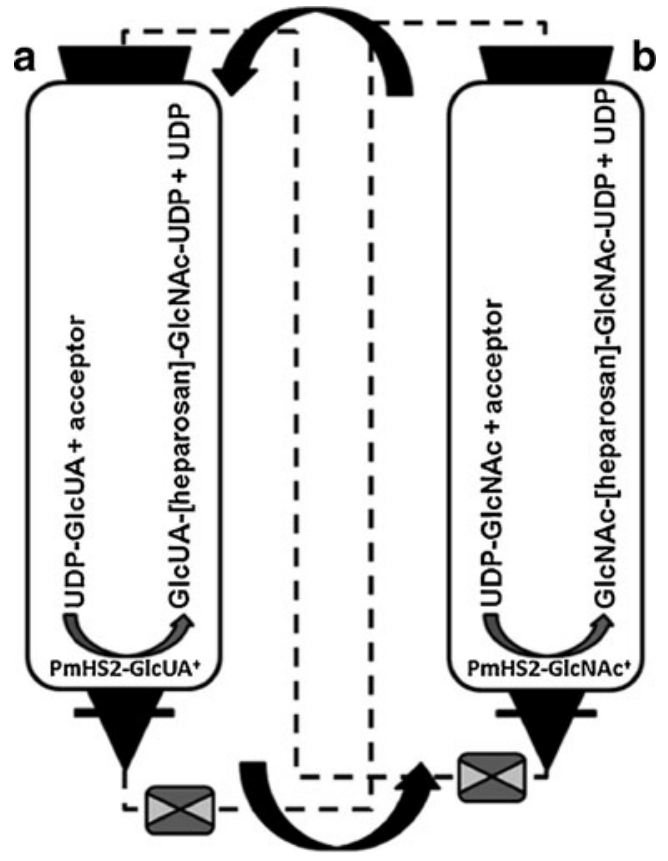

Fig. 1 Schematic representation of the procedure for the step-by-step elongation of heparosan oligosaccharides. The first step is the initiation of the heparosan chain (GlcUA-GlcNAc-UDP) by immobilized PmHS2-GlcUA ${ }^{+}$(column $A$ ) in the presence of UDP-GlcUA and UDP-GlcNAc, which was identified as the acceptor molecule (Chavaroche et al. 2011). Prior to be incubated with immobilized PmHS2-GlcNAc ${ }^{+}$(column B), the reaction mixture was heat treated $\left(65{ }^{\circ} \mathrm{C} / 15 \mathrm{~min}\right)$ to prevent residual enzyme activity. Successive incubation cycles of the reaction mixture (UDP-sugars and oligosaccharide acceptors [heparosan]-GlcNAc-UDP) enable to synthesize heparosan oligosaccharides of a defined chain length. The oligosaccharides still remain the UDP-group belonging to the first UDP-GlcNAc acceptor molecule as determined by MALDI-TOF MS analysis
UDP-GlcNAc for column A or B, respectively. The reaction mixtures were transferred from column A to column B in an alternating manner to elongate heparosan oligosaccharides to a defined polymerization degree. Each incubation step was performed for $3 \mathrm{~h}$ in the dark under orbital shaking conditions at $32{ }^{\circ} \mathrm{C}$. At the end of each incubation step, the reaction mixture was released from the column (e.g., column A) and was heat-inactivated $\left(15 \mathrm{~min}\right.$ at $\left.65^{\circ} \mathrm{C}\right)$ to avoid the possible presence of any residual enzyme activity before being loaded onto the other column (column B), vice versa. In addition, between each incubation cycle, the immobilized enzymes were washed with $2 \times 1 \mathrm{~mL}$ of Tris- $\mathrm{HCl}$ (40 mM, pH 7.15), $4 \mathrm{mM} \mathrm{Mn}^{2+}$, and $4 \mathrm{mM} \mathrm{Mg}^{2+}$.

\section{Analysis of the polymerization activity}

For the analysis of the polymerization activity, fresh immobilized and PD10-purified PmHS2 single-action transferases were used, and the same procedures as described before have been used (Chavaroche et al. 2010).

Coupled enzyme assay Samples were quenched by immersion in liquid nitrogen, and the UDP-sugars conversion was quantified by measuring the NADH reduction into $\mathrm{NAD}^{+}$at $340 \mathrm{~nm}$ in a coupled enzyme assay (Gosselin et al. 1994; Krupa et al. 2007).

Gel electrophoresis Heparosan oligomers/polymers synthesized during the enzymatic reactions were analyzed by Novex $20 \%$ Tris borate-EDTA (TBE) polyacrylamide gel (Invitrogen) and $2 \%(w / v)$ agarose gel electrophoresis. The sample preparation, the gel electrophoresis parameters, and the staining procedures were done according to Chavaroche et al. (2011). A selected hyaluronan molecular mass marker ranging from 27 to $495 \mathrm{kDa}$ (Select-HALoLadder, Hyalose) was used to estimate the heparosan molecular weight.

High-performance size exclusion chromatography analysis The size distribution and the molecular weight of the heparosan polymers were analyzed by HPSEC on an Ultimate 3000 (Dionex, Sunnyvale, USA) using three TosoH Bioscience TSK-gel columns in series $(4000,3000$, and 2500 Super AW, $150 \times 6.0 \mathrm{~mm}$ ) preceded by a TSK AWL guard column $(35 \times 4.6 \mathrm{~mm}$; TosoH Bioscience, Japan $)$ as previously described (Chavaroche et al. 2011). For each HPSEC analysis, the molecular weight and the size distribution of the samples were determined by fitting to a logarithmic model the elution time of the hyaluronan molecular mass markers (30, 160, and $262 \mathrm{kDa}$, Hyalose) (Chavaroche et al. 2010).

High-performance anion exchange chromatography analysis The composition of the reaction mixture (UDP- 
sugars and heparosan oligosaccharides) was analyzed by high-performance anion exchange chromatography (HPAEC) using an ICS-3000 Ion Chromatography HPLC system equipped with a CarboPac PA-1 column $(2 \times$ $250 \mathrm{~mm}$ ), in combination with a CarboPac PA guard column $(2 \times 25 \mathrm{~mm})$, a VWD-3100 single wave detector (Dionex, Sunnyvale, USA), and a pulsed electrochemical detector in pulsed amperometric detection mode (Dionex, Sunnyvale, CA, USA) in series. The same elution conditions as previously described were used (Chavaroche et al. 2011). The presence of a peak with UV detection, as with electrochemical detection, shows the presence of an UDP group (Orellana and Mohnen 1999).

Matrix-assisted laser desorption-ionization time-of-flight mass spectrometry analysis An Ultraflex workstation (Bruker Daltonics, Germany) equipped with a 337-nm laser was operated in the negative mode and calibrated with a mixture of peptide standards from Bruker Daltonics. Ions were accelerated with a $20-\mathrm{kV}$ voltage after a delayed extraction of 180 ns. Detection was performed using the reflector mode. Samples were ten times diluted in the matrix solution containing $10 \mathrm{mg} / \mathrm{mL}$ 2,5-dihydroxybenzoic acid in $50 \%(v / v)$ acetonitrile. For analysis, $2 \mu 1$ of the mixture was transferred to a matrix-assisted laser desorption-ionization (MALDI) sample plate and dried under a stream of warm air.

\section{Results}

The PmHS2 single-action transferases (PmHS2-GlcUA ${ }^{+}$and PmHS2-GlcNAc ${ }^{+}$) were immobilized on Ni-NTA agarose during the purification procedure. The effect of the immobilization on the polymerization activity and stability was determined first. The parameters influencing the PmHS2 polymerization process and the heparosan chain elongation were studied next. Therefore, the polymerization activity was monitored in the presence of non-equal amounts of immobilized PmHS2-GlcUA ${ }^{+}$and PmHS2-GlcNAc ${ }^{+}$, incubated together in the presence of variable UDP-sugar concentrations. A fractional factorial design was included to determine the influence of PmHS2-GlcUA ${ }^{+}$, PmHS2-GlcNAc ${ }^{+}$, UDPGlcUA, and UDP-GlcNAc concentrations. Furthermore, we used PmHS2-GlcUA ${ }^{+}$and PmHS2-GlcNAc ${ }^{+}$for step-by-step elongation of heparosan oligosaccharides in the absence of oligosaccharide templates.

Enzyme immobilization

By comparing the amount of PmHS2-GlcUA ${ }^{+} / \mathrm{PmHS}_{2}$ $\mathrm{GlcNAc}^{+}$protein immobilized onto Ni-NTA agarose and recovered after the PD10-purification step, it was found that per volume of induced $E$. coli culture, the amount of immobilized enzyme and PD10-purified enzymes were comparable based on the protein assay. It was determined that about $0.36 \mathrm{mg}$ of PmHS2 transferases was immobilized per $1 \mathrm{~mL}$ of Ni-NTA slurry (50:50\% agarose/buffer). The SDS-PAGE gel analysis of the fractions washed from the column after storage at $4^{\circ} \mathrm{C}$ revealed the presence of a small quantity of protein, suggesting protein leaching. Thus, in order to avoid possible contamination of each column by undesired PmHS2 enzyme activities, the reaction mixtures released from the columns were heat-inactivated for $15 \mathrm{~min}$ at $65^{\circ} \mathrm{C}$ before being applied from column A to column B and vice versa (Fig. 1).

The combined immobilized PmHS2 single-action transferases were more stable during storage at $4{ }^{\circ} \mathrm{C}$ than the combined PD10-purified single-action transferases (Fig. 2). The activity of the immobilized PmHS2 single-action transferases was stable during 2 days of storage. After 2 weeks of storage at $4{ }^{\circ} \mathrm{C}$, the immobilized PmHS2 single-action transferases exhibited about $75 \%$, and after 50 days of storage, they still exhibited about $60 \%$ of their initial activity. The PD10-purified single-action transferases were not stable at $4{ }^{\circ} \mathrm{C}$; after 1 day of storage, the activity decreased to about $50 \%$, and after 1 week, only $10 \%$ of the activity remained. The addition of ethylene glycol and metal ions $\left(\mathrm{MgCl}_{2}\right.$ and $\mathrm{MnCl}_{2}$ ) in the buffer (Tris- $\mathrm{HCl} 50 \mathrm{mM}, \mathrm{pH}$ 7.15) did not improve the enzyme stability of the PD10-purified singleaction transferases. Furthermore, immobilization did not hamper the polymerization process catalyzed by the PmHS2 single-action transferases. Indeed, the specific activity of the PmHS2 single-action transferase was comparable whether the enzymes were immobilized or PD10-purified. The polymerization activity of the PD10-purified PmHS2-

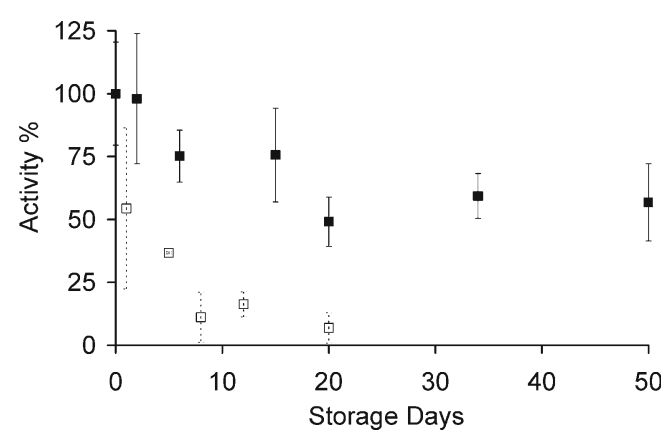

Fig. 2 Storage stability of immobilized and PD10-purified PmHS2 single-action transferases. The PmHS2 single-action transferases $(25-30 \mu \mathrm{g} / \mathrm{mL}$ of each transferase) were incubated together for $24 \mathrm{~h}$ in the presence of $5 \mathrm{mM}$ of each UDP-sugar (closed square immobilized and open square PD10-purified PmHS2 single-action transferases). The amount of UDP-sugar converted after $24 \mathrm{~h}$ of incubation at $32{ }^{\circ} \mathrm{C}$ was quantified by the coupled enzyme assay. The polymerization activity of the fresh immobilized and PD10purified enzymes $(t=0)$ was set as $100 \%$ 
$\mathrm{GlcUA}^{+}$and PmHS2-GlcNAc ${ }^{+}$, incubated together at $32{ }^{\circ} \mathrm{C}$ for 2 to $8 \mathrm{~h}$ in the presence of $5 \mathrm{mM}$ UDP-sugars, was about $0.065 \mathrm{nmol} / \mathrm{min} / \mu \mathrm{g}$ protein, similar as previously reported (Chavaroche et al. 2011). Under the same incubation condition, immobilized PmHS2 single-action transferases added together exhibited comparable catalytic efficiency, about $0.070 \mathrm{nmol} / \mathrm{min} / \mu \mathrm{g}$ protein.

Influence of each transferase domain on the polymerization activity

We previously reported that the heparosan chain elongation by PmHS2 is influenced by the UDP-sugar concentrations (Chavaroche et al. 2010, 2011). Here, in order to determine the influence of the PmHS2 transferases (glucuronyl transferase and $N$-acetylglucosamine transferase) on the heparosan initiation and elongation, the polymerization process of non-equal amount of immobilized PmHS2 single-action transferases (3 to $48 \mu \mathrm{g} / \mathrm{mL}$ of each transferase) was investigated when incubated with 1 or $5 \mathrm{mM}$ of each UDP-sugar. The polymerization activity observed in the presence of $12 \mu \mathrm{g} / \mathrm{mL}$ of each PmHS2 transferase (PmHS2-GlcUA ${ }^{+}$ and PmHS2-GlcNAc ${ }^{+}$) was used as a reference (Figs. 3 and 4).

When incubated in the presence of PmHS2 single-action transferase ratio below 1 ( 3 to $6 \mu \mathrm{g} / \mathrm{mL} \mathrm{E1} \mathrm{and} 12 \mu \mathrm{g} / \mathrm{mL}$ E2), the polymerization process was hampered resulting in a low polymerization activity (Fig. 3). In the presence of low concentrations PmHS2-GlcUA ${ }^{+}$or PmHS2-GlcNAc ${ }^{+}$, it is likely that both the initiation and the chain elongation of heparosan polymers are hindered, resulting in the synthesis of heparosan chain concentrations below the detection limit of the agarose gels (Fig. 4).

When the reaction mixtures were incubated in the presence of PmHS2 single-action transferase ratio above 1 $(\mathrm{E} 1 / \mathrm{E} 2>1)$, the polymerization process was not hampered, but the ratio of transferases had different effects on the overall polymerization process. In the presence of an excess of PmHS2-GlcUA ${ }^{+}$(from 12 up to $48 \mu \mathrm{g} / \mathrm{mL}$ ) and $1 \mathrm{mM}$ of each UDP-sugar, the polymerization efficiency increased proportionally to the increase of PmHS2-GlcUA ${ }^{+}$concentration (Fig. 3a). Indeed, in the presence of $36 \mu \mathrm{g} / \mathrm{mL}$ PmHS2-GlcUA ${ }^{+}$and $12 \mu \mathrm{g} / \mathrm{mL}$ PmHS2-GlcNAc ${ }^{+}$, the polymerization efficiency increased 2.5 times in comparison to the efficiency obtained in the presence of equal amounts of PmHS2-GlcUA ${ }^{+}$and PmHS2-GlcNAc ${ }^{+}$. The polymerization efficiency also increased proportionally to the increase of PmHS2-GlcUA ${ }^{+}$concentration in the presence of $5 \mathrm{mM}$ of each UDP-sugar (Fig. 3b). However, it was observed that for high PmHS2-GlcUA ${ }^{+}$concentrations (36 to $48 \mu \mathrm{g} / \mathrm{mL}$ ) and a PmHS2-GlcNAc ${ }^{+}$concentration of $12 \mu \mathrm{g} / \mathrm{mL}$, the polymerization efficiency leveled off.
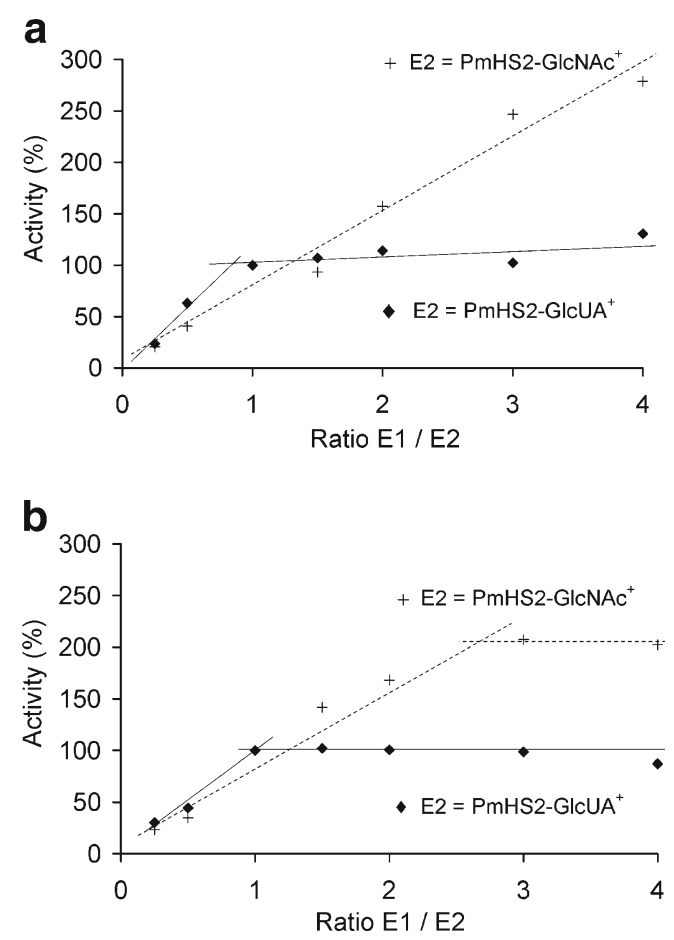

Fig. 3 Influence of each PmHS2 single-action transferase on the overall polymerization activity. Non-equal amount of freshly immobilized PmHS2-GlcUA ${ }^{+}$and PmHS2-GlcNAc ${ }^{+}$(ranging from 3 to 48 $\mu \mathrm{g} / \mathrm{mL}$ ) were mixed together and were incubated for $4 \mathrm{~h}$ in the presence of $1 \mathrm{mM}$ (a) and $5 \mathrm{mM}$ (b) of each UDP-sugar (UDP-GlcUA and UDP-GlcNAc). The amount of UDP-sugars converted after $4 \mathrm{~h}$ of incubation was determined by the coupled enzyme assay. The polymerization activity observed in the presence of equal amount of PmHS2-GlcUA ${ }^{+}$and PmHS2-GlcNAc ${ }^{+}(12 \mu \mathrm{g} / \mathrm{mL}$ of each transferase, $\mathrm{E} 1 / \mathrm{E} 2=1$ ) was set at $100 \%$ relative activity. In all the experiments, E2 was set as a constant $(12 \mu \mathrm{g} / \mathrm{mL})$ and $\mathrm{E} 1$ varied from 3 to $48 \mu \mathrm{g} / \mathrm{mL}$. These results have been obtained from triplicate experiments; the standard deviation was $\pm 3-10 \%$

Agarose gel electrophoresis analysis of the reaction mixtures (PmHS2-GlcUA ${ }^{+} / \mathrm{PmHS}-\mathrm{GlcNAc}^{+}$) in the presence of either 1 or $5 \mathrm{mM}$ of each UDP-sugar showed that increasing amounts of PmHS2-GlcUA ${ }^{+}$(below $36 \mu \mathrm{g} / \mathrm{mL}$ ) resulted in the synthesis of heparosan chains with similar molecular weight and the same size distribution (Fig. 4a). However, in the presence of $5 \mathrm{mM}$ of each UDP-sugar in the reaction mixture, compared to $1 \mathrm{mM}$ of UDP-sugars, a higher number of heparosan chains were synthesized as shown with the coupled enzyme assay. This suggests that despite the higher number of chains to elongate the incorporation of GlcNAc and GlcUA, residues occurred at the same velocity for each heparosan chain. Nevertheless, heparosan polymers with a lower molecular weight were synthesized in the presence of $5 \mathrm{mM}$ UDP-sugars and 4-fold more the amount of PmHS2-GlcUA ${ }^{+}(48 \mu \mathrm{g} / \mathrm{mL})$ than PmHS2-GlcNAc ${ }^{+}(12 \mu \mathrm{g} / \mathrm{mL})$. Probably, the elevated number of heparosan chains initiated by PmHS2-GlcUA ${ }^{+}$ either inhibited or saturated PmHS2-GlcNAc ${ }^{+}$, resulting in 
Fig. 4 Agarose gel of heparosan polymers synthesized in the presence of non-equal amount of immobilized PmHS2 single-action transferases. The reaction mixtures were incubated for $4 \mathrm{~h}$ in the presence of $5 \mathrm{mM}$ of each UDP-sugar (UDP-GlcUA and UDP-GlcNAc) and non-equal concentration of PmHS2-

$\mathrm{GlcUA}^{+}$and PmHS2-GlcNAc ${ }^{+}$

(E1/E2 ratio ranging from 0.25 to 4 , in which $\mathrm{E} 1=3$ to $48 \mu \mathrm{g} /$ $\mathrm{mL}$ and $\mathrm{E} 2=12 \mu \mathrm{g} / \mathrm{mL}$, respectively)

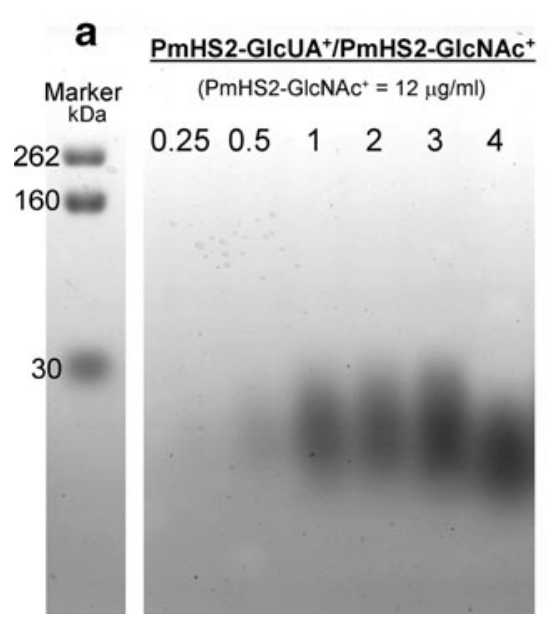

b

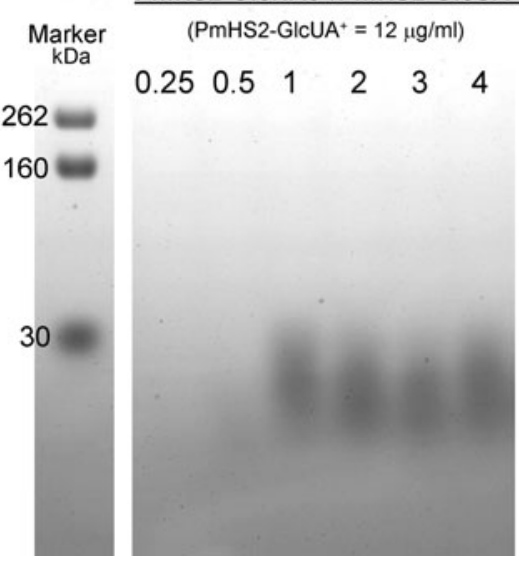

the incapability of the PmHS2-GlcNAc ${ }^{+}$to elongate all the chains at the same velocity.

When PmHS2-GlcNAc ${ }^{+}$was added in excess to the incubation mixtures (from 12 to $48 \mu \mathrm{g} / \mathrm{mL}$ ), the polymerization efficiency did not increase (Fig. 3). The same observation was made in reaction mixtures incubated with 1 or $5 \mathrm{mM}$ of each UDP-sugar. Agarose gel electrophoresis analysis and the coupled enzyme assay confirmed that independently of the PmHS2-GlcNAc ${ }^{+}$ concentrations (from 12 to $48 \mu \mathrm{g} / \mathrm{mL}$ ), the same number of heparosan chains were initiated and elongated to the same length (Fig. 4b). These results suggest that the PmHS2-GlcUA ${ }^{+}$activity is limiting the overall polymerization efficiency.

Experimental design analysis

To be able to understand and to control heparosan polymer elongation using PmHS2 glycosyltransferases, the effect of combined parameters on PmHS2 polymerization process were investigated using a fractional factorial design with four variables (three levels $\left(3^{4-1}\right)$ for each variable). Thus, 27 reaction mixtures were prepared in which different combinations of UDP-sugar, and PmHS2 single-action transferase concentrations were added (Supplementary information Table 1). The reaction mixtures were analyzed by the coupled enzyme assay to monitor the polymerization efficiency (data not shown), and they were also analyzed by HPSEC to assess the polymer elongation process.

Using the Mallow's $\mathrm{Cp}$ criterion, the analysis of the 27 reaction mixtures showed that eight parameters $\left(R^{2} 0.848\right)$ were needed to obtain the best fitting model. The following equation describes the influence of the single-action transferase concentrations and UDP-sugar concentrations on the heparosan elongation process:

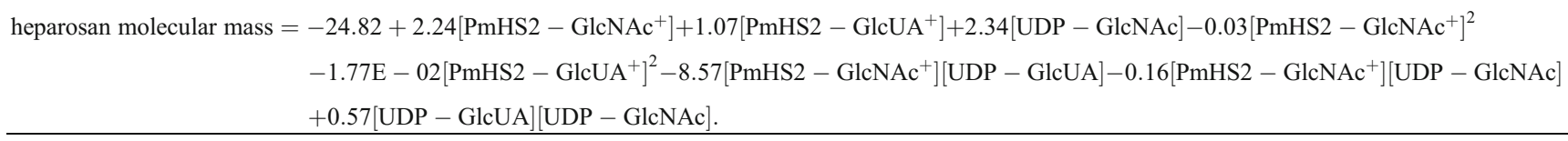

This model showed that the four variables (PmHS2GlcUA $^{+}$, PmHS2-GlcNAc ${ }^{+}$, UDP-GlcUA, and UDPGlcNAc concentrations) influenced the polymerization activity with respect to the heparosan molecular weight. It was found that only the PmHS2-GlcNAc ${ }^{+}\left(x_{1}\right)$, PmHS2-GlcUA $^{+}\left(x_{2}\right)$, and UDP-GlcNAc $\left(x_{4}\right)$ concentrations are represented as main effect. In addition, the coefficient for the PmHS2-GlcNAc ${ }^{+}$concentration is higher than for the PmHS2-GlcUA ${ }^{+}$concentration. Despite the fact that the UDP-GlcUA concentration $\left(x_{3}\right)$ was not represented as a main effect, the interaction effects showed that UDP-GlcUA concentration influences the heparosan elongation. The analysis of the contour plot visualization obtained from this analysis is presented in Fig. 5.

In reaction mixtures incubated with increasing concentrations of PmHS2-GlcUA ${ }^{+}$(7, 21, or $\left.34 \mu \mathrm{g} / \mathrm{mL}\right)$ and a constant concentration of PmHS2-GlcNAc ${ }^{+}$(Fig. 5, from left to right), it was observed that the polymer molecular weight was neither influenced by the UDP-sugar concentration nor by the PmHS2-GlcUA ${ }^{+}$concentrations (Fig. 5a-c). Nevertheless, the increase of PmHS2-GlcUA ${ }^{+}$concentration resulted in the synthesis of a higher number of heparosan chains and higher polymerization efficiency, as observed 


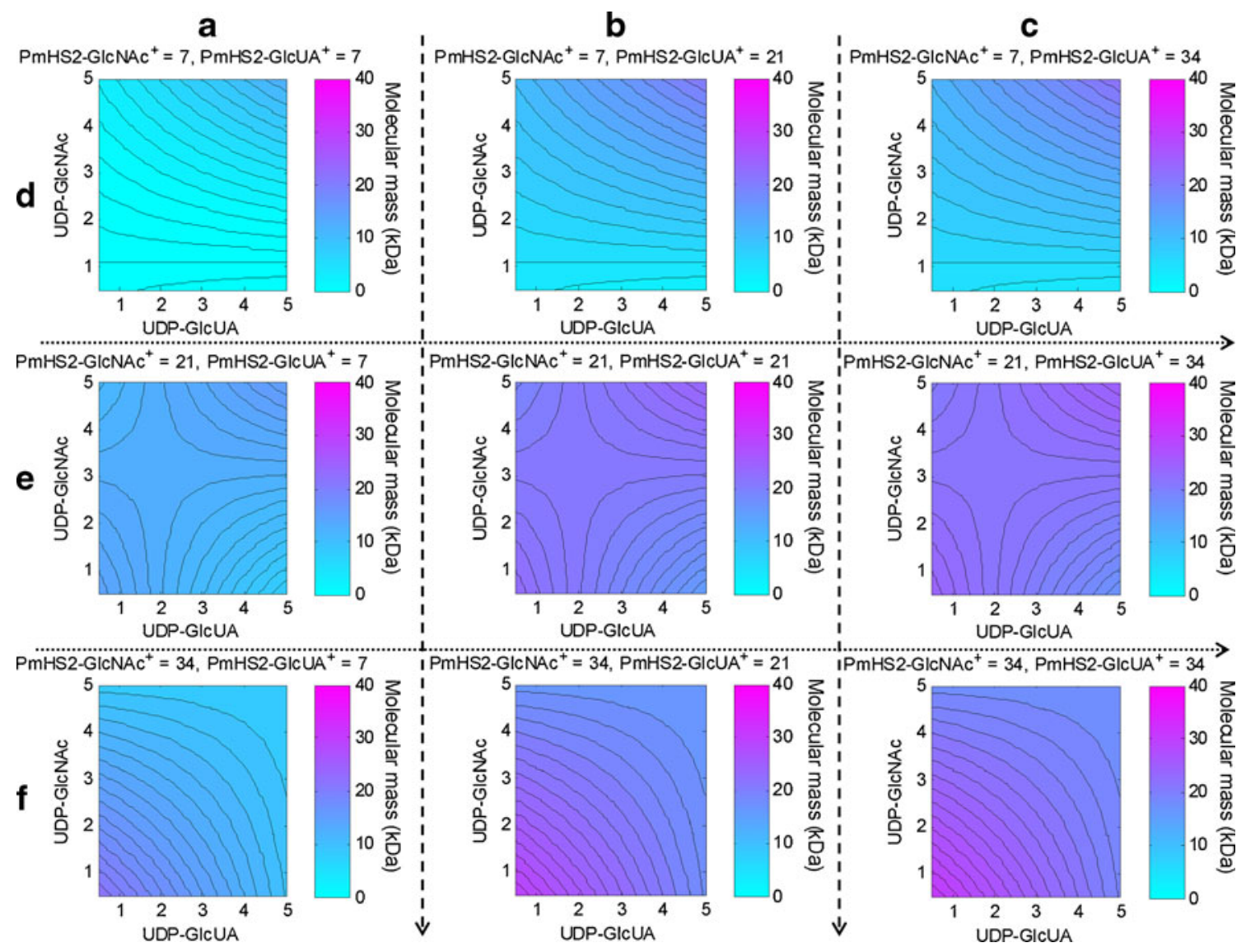

Fig. 5 Contour plot visualization of the statistical analysis of the PmHS2 single-action transferase polymerization activity with respect to the polymer molecular weight. The reaction mixtures were prepared according to a fractional factorial design with four variables and three levels $\left(3^{4}-1\right)$ for each variable (immobilized PmHS2-GlcUA ${ }^{+}$and immobilized PmHS2-GlcNAc ${ }^{+}$(micrograms per milliliter), UDP-
GlcUA, and UDP-GlcNAc (millimolars)). After $4 \mathrm{~h}$ of incubation at $32^{\circ} \mathrm{C}$, the heparosan average molecular mass (kilodaltons, presented in the scale at the right of each graph) was determined by highperformance size exclusion chromatography. The data were visualized using the mathematical software package Matlab using the coupled enzyme assay (data not shown). In the presence of a low PmHS2-GlcUA ${ }^{+}$concentration $(7 \mu \mathrm{g} / \mathrm{mL})$, the polymerization was hampered as it can be assessed by the blue color trend (Fig. 5a); this is probably due to the low number of heparosan chain initiated.

In the reaction mixtures incubated in the presence of increasing PmHS2-GlcNAc ${ }^{+}$concentrations (7, 21, or $34 \mu \mathrm{g} /$ $\mathrm{mL}$ ) in combination with a constant concentrations of PmHS2-GlcUA ${ }^{+}$(Fig. 5, from top to bottom) resulted in different polymer elongation profiles as function of the UDP-sugar concentration present in the reaction mixture (Fig. 5d-f). A constant concentration of PmHS2-GlcUA ${ }^{+}$in the reaction mixture implies the initiation of the same number of heparosan chains at a given UDP-sugar concentration. Thus, the polymerization trend observed is mainly the result of the PmHS2-GlcNAc ${ }^{+}$concentration. In the presence of a low concentration of PmHS2-GlcNAc ${ }^{+}(7 \mu \mathrm{g} / \mathrm{mL})$, the longest heparosan polymers were synthesized when incubated with high UDP-sugar concentrations (Fig. 5d). The fact that the polymerization occurred in the presence of a high UDPsugar concentration suggests that PmHS2-GlcNAc ${ }^{+}$required a high concentration of heparosan oligosaccharides to perform its catalytic activity. Therefore, it was assumed that the affinity of the GlcNAc acceptor site is low. For the intermediate concentration of PmHS2-GlcNAc ${ }^{+}(21 \mu \mathrm{g} /$ $\mathrm{mL}$ ), it seemed that the synthesis of long heparosan polymers was favored in the presence of either high or low equimolar UDP-sugar concentrations (Fig. 5e), while the production of long heparosan chains and the polymerization activity was hampered in the presence of low UDP-GlcNAc concentrations combined with high UDP-GlcUA concentrations, incubation conditions which were determined to be not suitable to initiate heparosan chains (Chavaroche et al. 2011). In the presence of high PmHS2-GlcNAc ${ }^{+}(34 \mu \mathrm{g} / \mathrm{mL})$ concentrations, the longest polymers were obtained when incubated in the presence of low UDP-sugar concentration (Fig. 5f). Low UDP-sugar concentrations resulted in 
the synthesis of a lower number of heparosan chains (Chavaroche et al. 2010), which may avoid the saturation of the PmHS2-GlcNAc ${ }^{+}$acceptor site and thus favor the elongation process.

Step-by-step elongation of heparosan oligosaccharides

It was observed that PmHS2 polymerization process is influenced by both transferase activities and both UDPsugar concentrations, rendering the control of heparosan chain elongation difficult. PmHS2-GlcUA ${ }^{+}$was reported to initiate heparosan chains when incubated in the presence of both UDP-sugars (Chavaroche et al. 2011). This implies that PmHS2-GlcUA $^{+}$is active without forming a protein complex with PmHS2-GlcNAc ${ }^{+}$. To determine if PmHS2$\mathrm{GlcNAc}^{+}$is also active as a single function transferase and thus to evaluate if the PmHS2-single-action transferases could be used to elongate step-by-step heparosan oligosaccharides in the absence of template molecules, PmHS2$\mathrm{GlcUA}^{+}$and PmHS2-GlcNAc${ }^{+}$were immobilized on Ni-NTA resin and loaded in separate columns. In order to control the elongation of heparosan oligosaccharides, the reaction mixtures (buffer, UDP-sugars, and oligosaccharides) were recycled from one column (A) to the other (B) (Fig. 1). The analysis of the reaction mixture using HPAEC, MALDI-time-of-flight mass spectrometry (TOF MS), and $20 \%$ TBE gel electrophoresis (data not shown) showed that

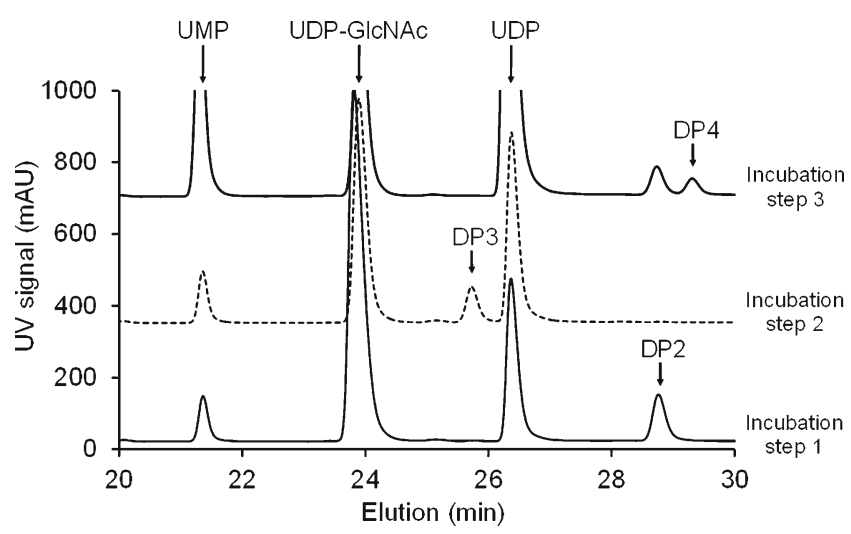

Fig. 6 High-performance anion exchange chromatography analysis of the reaction mixtures obtained after different incubation steps in the presence of immobilized PmHS2-GlcUA ${ }^{+}$or PmHS2-GlcNAc ${ }^{+}$. Heparosan disaccharides (DP2) were released from the PmHS2-GlcUA ${ }^{+}$ column (column A; $0.5 \mathrm{mg}$ PmHS2-GlcUA ${ }^{+} / \mathrm{mL}$ reaction, incubation step 1), heparosan trisaccharide (DP3) from the PmHS2-GlcNAc ${ }^{+}$ column (column B; $0.5 \mathrm{mg} \mathrm{PmHS} 2-\mathrm{GlcNAc}^{+} / \mathrm{mL}$ reaction, incubation step 2), and a mixture of heparosan disaccharides (DP2) and tetrasaccharides (DP4) from the PmHS2-GlcUA ${ }^{+}$column (incubation step 3). UMP, UDP-GlcNAc, UDP, and UDP-GlcUA (not shown) eluted after $21.1,23.5,26.0$, and $33.6 \mathrm{~min}$, respectively. The annotation $\mathrm{DP}_{X}$ stands for "degree of polymerization $X$," in which $X$ represents the number of monosaccharide units heparosan oligosaccharides with a defined length could be synthesized by the PmHS2 single-action transferases in the absence of oligosaccharide templates. The monitoring of the reaction mixtures after each incubation step by HPAEC and MALDI-TOF MS showed that the oligosaccharides were completely converted after each incubation steps (Figs. 6 and 7). As can be observed with HPAEC (Fig. 6), the disaccharides (DP2) synthesized during the "step 1" incubation, in the presence of PmHS2-GlcUA (column A), were totally converted into trisaccharides (DP3) at the end of the "step 2" incubation in the presence of PmHS2$\mathrm{GlcNAc}^{+}$(column B). Analysis of the "step 2" reaction mixture by MALDI-TOF MS showed the absence of disaccharides and confirmed the total conversion of the DP2 into DP3 by PmHS2-GlcNAc ${ }^{+}$. At the end of the "step 3" incubation with PmHS2-GlcUA ${ }^{+}$only, disaccharides (DP2) and tetrasaccharides (DP4) heparosan were observed by both HPAEC and MALDI-TOF MS analysis.

Analysis of the oligosaccharide content (HPAEC and MALDI-TOF MS) of the different reaction mixtures (step 3 to step 9) showed that the incubation in the presence of PmHS2-GlcNAc ${ }^{+}$resulted only in odd numbered oligosaccharides (DP3, DP5, and DP7), while even numbered oligosaccharides (DP2, DP4, DP6, and DP8) were found only after incubation with PmHS2-GlcUA ${ }^{+}$(Fig. 7). The presence of only odd numbered oligosaccharides in the PmHS2$\mathrm{GlcNAc}^{+}$mixtures is due to the fact that PmHS2-GlcNAc ${ }^{+}$ is not capable of initiating the elongation of heparosan and thus cannot synthesize even numbered oligosaccharides. The presence of heparosan oligosaccharides with different elongation degree showed that the initiation of new chains occurred during each incubation step with PmHS2-GlcUA ${ }^{+}$.

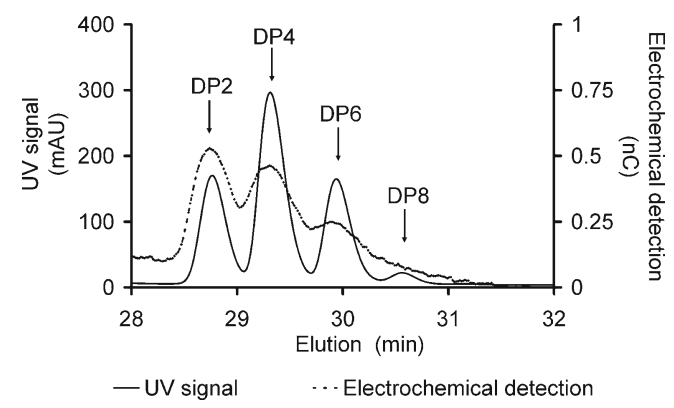

Fig. 7 High-performance anion exchange chromatography analysis of the reaction mixture released from the PmHS2-GlcUA ${ }^{+}$column (column A). Even numbered heparosan oligosaccharide mixture from disaccharides up to octasaccharides was synthesized by the immobi-

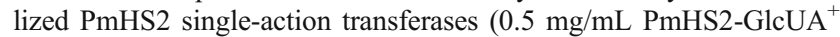
and $0.5 \mathrm{mg} / \mathrm{mL}$ PmHS2-GlcNAc ${ }^{+}$), as shown in Fig. 1. The reaction mixture was obtained after nine successive incubation cycles (last incubation cycle on column A). The annotation $\mathrm{DP}_{X}$ stands for "degree of polymerization $X$ ", in which $X$ represents the number of monosaccharide units 
Residual amount of UDP-GlcNAc in the reaction mixture is probably responsible for the initiation of new chains. After nine successive incubation cycles in PmHS2-GlcUA ${ }^{+}$and PmHS2-GlcNAc ${ }^{+}$columns, the reaction mixture released from PmHS2-GlcUA ${ }^{+}$column showed that only even numbered heparosan oligosaccharides (disaccharides (DP2) to octasaccharides (DP8)) were produced (Fig. 7). The disaccharides GlcUA-GlcNAc-UDP (DP2) eluted after $28.8 \mathrm{~min}$, and the other heparosan oligosaccharides of DP4, DP6, and DP8 eluted after 29.4, 30.0, and $30.6 \mathrm{~min}$, respectively. The analysis of the reaction mixtures by MALDI-TOF MS showed that the UDP group still remained attached to the growing oligosaccharide chains (DP2 $\mathrm{m} / \mathrm{z}$ 782.6, DP4 $\mathrm{m} / \mathrm{z}$ 1,160.3, DP6 $m / z$ 1,539.0, and DP8 $m / z ~ 1,918.0$ ).

\section{Discussion}

In this report, we showed that PmHS2-GlcUA ${ }^{+} / \mathrm{PmHS}_{2}-$ $\mathrm{GlcNAc}^{+}$were successfully immobilized on Ni-NTA agarose during the purification step. The polymerization activity of freshly PD10-purified and freshly immobilized PmHS2 single-action transferases was found to be similar, and it was shown that immobilization did not have any effect on the polymerization activity. The stability of the combined single-action transferases increased when stored at $4{ }^{\circ} \mathrm{C}$. The single-action transferases have not been studied separately, and the decrease of the stability could be due to only one of the two single-action transferases.

Previously it has been shown that the single-action transferases can be used as a model to describe dual transferase PmHS2 activity (Chavaroche et al. 2011); thus, immobilized PmHS2 single-action transferases can also be used to study PmHS2 polymerization mechanism. The use of IMAC such as Ni-NTA agarose is frequently reported for the purification of protein, but its usage for immobilization purpose is not common (Liu et al. 2002). Here we showed that it is a suitable method to study PmHS2 transferases.

Influence of each transferase domains on the polymerization activity. The PmHS2 polymerization process was investigated in the presence of different ratios of PmHS2$\mathrm{GlcUA}^{+}$and PmHS2-GlcNAc${ }^{+}$. It was found that both transferase domains do not exhibit the same catalytic efficiency.

Here, we report that the addition of an excess of PmHS2$\mathrm{GlcUA}^{+}$in comparison to PmHS2-GlcNAc${ }^{+}$increased the polymerization activity, while an excess of PmHS2$\mathrm{GlcNAc}^{+}$did not influence the catalytic efficiency. The results suggest that the glucuronyl transferase (PmHS2$\mathrm{GlcUA}^{+}$) is the limiting catalyst in the overall PmHS2 polymerization process, either by controlling the amount of chain initiated and by exhibiting per heparosan chain a slow and a constant rate in transferring GlcUA residue in comparison to the GlcNAc transfer by PmHS2-GlcNAc ${ }^{+}$. We previously reported that for a same amount of protein, the specific activity of PmHS2-single-action transferases was 2-fold lower than for PmHS2 (Chavaroche et al. 2011). The results obtained now certainly indicate that PmHS2 single-action transferases exhibit similar polymerization efficiency as PmHS2. Indeed, the difference of activity previously observed might have been due to the fact that with comparable amounts of PmHS2-single-action transferases and PmHS2, there is twice less active PmHS2$\mathrm{GlcUA}^{+}$in the reaction mixture of PmHS2-single-action transferases compared to the one with PmHS2. It was also observed that $N$-acetylglucosaminyl transferase (PmHS2$\mathrm{GlcNAc}^{+}$) is either saturated or inhibited in the presence of a too high number of chains. This may explain why PmHS2 synthesizes low molecular weight heparosan polymers in the presence of high UDP-sugars concentrations (Chavaroche et al. 2010).

In addition, the analysis of the experimental design showed that the four variables: PmHS2-GlcUA ${ }^{+}$, PmHS2$\mathrm{GlcNAc}^{+}$, UDP-GlcUA, and UDP-GlcNAc concentrations influence the heparosan elongation. This is in agreement with what has been reported previously for the PmHS2 polymerization process (Chavaroche et al. 2010, 2011). It was observed that the polymer chain length and size distribution is mainly determined by the number of chain initiated which depends on the concentration of UDP-sugars (Chavaroche et al. 2010), on the number of acceptor molecules (UDP-GlcNAc) (Chavaroche et al. 2011), and on the number of acceptor sites (PmHS2-GlcUA ${ }^{+}$). The heparosan chain length is also influenced by the catalytic efficiency of the transferases to elongate heparosan chains. The PmHS2$\mathrm{GlcNAc}^{+}$was found to play a critical role in the heparosan chain length, by being saturated or inhibited in the presence of a too high number of chains. These results correlate with previously observed results when the dual action transferase PmHS2 was incubated with high concentration of UDPsugars (Chavaroche et al. 2010).

Also, for the first time, we showed that PmHS2 singleaction transferases are independent and do not need complex formation with each other to exhibit activity. This represents a great advantage over the mammalians heparosan synthases (EXT1 and EXT2) (Mccormick et al. 2000; Kim et al. 2003) and the E. coli heparosan synthases (KfiC and KfiA) (Griffiths et al. 1998; Hodson et al. 2000; Sugiura et al. 2010) to control the elongation of heparosan.

For the first time, PmHS2 single-action transferases have been used to elongate step-by-step heparosan oligosaccharides. It was observed for each incubation step that the heparosan oligosaccharides were completely elongated. Since only PmHS2-GlcUA ${ }^{+}$is capable to initiate heparosan chains (Chavaroche et al. 2011), this resulted in the presence of only even numbered heparosan oligosaccharides when 
incubated with PmHS2-GlcUA ${ }^{+}$and only odd numbered oligosaccharides with PmHS2-GlcNAc ${ }^{+}$. HPAEC analysis revealed that the odd numbered oligosaccharides (nonreducing end GlcNAc) eluted earlier than the even numbered oligosaccharides (non-reducing end GlcUA). The trisaccharide GlcNAc-GlcUA-GlcNAc-UDP eluted at $25.8 \mathrm{~min}$, while the disaccharide GlcUA-GlcNAc-UDP eluted at $28.8 \mathrm{~min}$. This kind of elution behavior has been reported before for hyaluronan oligosaccharides (Price et al. 1997; Blundell and Almond 2006). The analysis of the reaction mixtures by MALDI-TOF MS showed that the UDP group remained attached to the growing oligosaccharide chains. The effect of the UDP-group during medical application should be evaluated, and if needed, an additional step leading to the UDP removal should be implemented.

The controlled synthesis of GAGs has not been reported yet in the absence of template (Deangelis et al. 2003; Sugiura et al. 2008; Liu et al. 2010). This approach is of interest since the production of template molecules is expensive and laborious. Production of templates either by chemical hydrolysis (Sismey-Ragatz et al. 2007) or enzymatic degradation (Ernst et al. 1995) of heparosan K5 polysaccharides is not a well-controlled process, and thus to obtain monodisperse heparosan molecules, a fractionation step is required. In addition, heparin lyases cleave the glycosidic bond via an elimination reaction resulting in the formation of oligosaccharides containing unsaturated uronic acid residues at the non-reducing end terminus. In order to use the templates for polymerization, the saturated uronic acid residues need to be removed using mercuric salts or by ozonolysis (Ludwigs et al. 1987; Masuko et al. 2011). Synthesis of heparosan disaccharides from nucleotide sugars requires the incubation of PmHS2-GlcUA ${ }^{+}$in the presence of both UDP-sugars (Chavaroche et al. 2011). The production of a mixture of heparosan oligosaccharides is due to the initiation of new heparosan chains for each elongation step with PmHS2-GlcUA ${ }^{+}$. This shows that both UDP-GlcNAc and UDP-GlcUA are present in the reaction mixture. Thus, to synthesize monodisperse heparosan oligosaccharides, the incubation conditions enabling to reach the complete conversion of the UDP-GlcNAc by PmHS2GlcNAc $^{+}$should be determined to avoid the presence of both UDP-sugars when incubated with PmHS2-GlcUA ${ }^{+}$. Furthermore, at the end of the second incubation step, the purification of the heparosan trisaccharides to be used as templates could facilitate the production of monodisperse heparosan polymers during the step-by-step elongation.

This research describes for the first time the ability to elongate heparosan oligosaccharides step by step using single-action heparosan transferases from $P$. multocida. Due to the uncontrolled hydrolysis of UDP-sugar, a precise quantitation was not possible. The reactions should be optimized (at this moment on microgram scale) in order to increase the yield of oligosaccharide synthesized per amount of substrate. By improving all the aspects mentioned above, higher yields of enzymatically synthesized well-defined heparosan chain length should be possible.

To summarize, we found that both UDP-sugars and both heparosan transferases influence the polymerization with respect to the catalytic efficiency and the polymer molecular weight. The glucuronyl transferase (PmHS2-GlcUA ${ }^{+}$) catalytic activity is limiting the overall polymerization process activity, and the $\mathrm{N}$-acetylglucosaminyl transferase (PmHS2$\mathrm{GlcNAc}^{+}$) influences the polymer elongation depending on the number of chains to elongate and the UDP-sugar concentrations present in the reaction mixture. A more detailed analysis of the kinetic parameters should help to further optimize the polymerization conditions to control heparosan chain length and size distribution. We also showed, for the first time, that PmHS2 single-action transferases incubated separately in the absence of template molecules are capable to elongate step-by-step heparosan oligomers. PmHS2$\mathrm{GlcUA}^{+}$and PmHS2-GlcNAc ${ }^{+}$are not only independent transferases, but they can also use modified UDP-sugars to elongate heparosan (Sismey-Ragatz et al. 2007; Liu et al. 2010). These properties are of interest to facilitate the in vitro synthesis of Hep/HS-like products (Liu et al. 2010) and to enlarge the potential of heparin and heparan sulfate biological activity (Sismey-Ragatz et al. 2007). In the future, more research is needed to produce heparosan oligosaccharides with a defined chain length and in high quantity in order to determine their exact biological properties in in vitro and in vivo experiments.

Acknowledgments The authors would like to thank Eric Boer for his valuable help and advice on the fractional factorial design. This project is carried out in close collaboration with "MSD, Oss" and financially supported by the Netherlands Ministry of Economic Affairs and the B-Basic partner organizations (www.b-basic.nl) through B-Basic, a public-private NWO-ACTS program (ACTS, Advanced Chemical Technologies for Sustainability).

Open Access This article is distributed under the terms of the Creative Commons Attribution Noncommercial License which permits any noncommercial use, distribution, and reproduction in any medium, provided the original author(s) and source are credited.

\section{References}

Bellaiche Y, The I, Perrimon N (1998) Tout-velu is a Drosophila homologue of the putative tumour suppressor EXT-1 and is needed for Hh diffusion. Nature 394:85-88

Bishop JR, Schuksz M, Esko JD (2007) Heparan sulphate proteoglycans fine-tune mammalian physiology. Nature 446:1030-1037

Blundell CD, Almond A (2006) Enzymatic and chemical methods for the generation of pure hyaluronan oligosaccharides with both odd and even numbers of monosaccharide units. Anal Biochem 353:236-247 
Box GEP, Draper NR (1987) Empirical model building and response surfaces. Wiley, New York

Busse M, Kusche-Gullberg M (2003) In vitro polymerization of heparan sulfate backbone by the EXT proteins. J Biol Chem 278:41333-41337

Chavaroche AAE, Springer J, Kooy F, Boeriu C, Eggink G (2010) In vitro synthesis of heparosan using recombinant Pasteurella multocida heparosan synthase PmHS2. Appl Microbiol Biotechnol 85:1881-1891

Chavaroche AAE, Van Den Broek LAM, Springer J, Boeriu C, Eggink G (2011) Analysis of the polymerization initiation and activity of Pasteurella multocida heparosan synthase PmHS2, an enzyme with glycosyltransferase and UDP-sugar hydrolase activity. J Biol Chem 286:1777-1785

Chen Y, Maguire T, Hileman R, Esko J, Linhardt R, Marks R (1997) Dengue virus infectivity depends on envelope protein binding to target cell heparan sulfate. Nat Med 3:866-871

Chen J, Jones CL, Liu J (2007) Using an enzymatic combinatorial approach to identify anticoagulant heparan sulfate structures. Chem Biol 14:986-993

Deangelis PL, Oatman LC, Gay DF (2003) Rapid chemoenzymatic synthesis of monodisperse hyaluronan oligosaccharides with immobilized enzyme reactors. J Biol Chem 278:35199-35203

Ernst S, Langer R, Cooney C, Sasisekharan R (1995) Enzymatic degradation of glycosaminoglycans. Crit Rev Biochem Mol Biol 30:387-444

Gosselin S, Alhussaini M, Streiff MB, Takabayashi K, Palcic MM (1994) A continuous spectrophotometric assay for glycosyltransferases. Anal Biochem 220:92-97

Griffiths G, Cook NJ, Gottfridson E, Lind T, Lidholt K, Roberts IS (1998) Characterization of the glycosyltransferase enzyme from the Escherichia coli $\mathrm{K} 5$ capsule gene cluster and identification and characterization of the glucuronyl active site. J Biol Chem 273:11752-11757

Hodson N, Griffiths G, Cook N, Pourhossein M, Gottfridson E, Lind T, Lidholt K, Roberts IS (2000) Identification that KfiA, a protein essential for the biosynthesis of the Escherichia coli $\mathrm{K} 5$ capsular polysaccharide, is an alpha-UDP-GlcNAc glycosyltransferase. The formation of a membrane-associated $\mathrm{K} 5$ biosynthetic complex requires KfiA, KfiB, and KfiC. J Biol Chem 275:27311-27315

Izumikawa T, Egusa N, Taniguchi F, Sugahara K, Kitagawa H (2006) Heparan sulfate polymerization in Drosophila. J Biol Chem 281:1929-1934

Kane TA, White CL, Deangelis PL (2006) Functional characterization of PmHS1, a Pasteurella multocida heparosan synthase. J Biol Chem 281:33192-33197

Kim BT, Kitagawa H, Tanaka J, Tamura JI, Sugahara K (2003) In vitro heparan sulfate polymerization. J Biol Chem 278:41618-41623

Krupa JC, Shaya D, Chi L, Linhardt RJ, Cygler M, Withers SG, Mort JS (2007) Quantitative continuous assay for hyaluronan synthase. Anal Biochem 361:218-225

Laremore TN, Zhang F, Dordick JS, Liu J, Linhardt RJ (2009) Recent progress and applications in glycosaminoglycan and heparin research. Curr Opin Chem Biol 13:633-640

Lazo-Langner A, Goss GD, Spaans JN, Rodger MA (2007) The effect of low-molecular-weight heparin on cancer survival. A systematic review and meta-analysis of randomized trials. J Thromb Haemost 5:729-737

Lindahl U (2007) Heparan sulfate-protein interactions - a concept for drug design? Thromb Haemost 98:109-115

Linhardt RJ, Toida T (2004) Role of glycosaminoglycans in cellular communication. Acc Chem Res 37:431-438

Liu Z, Zhang J, Chen X, Wang PG (2002) Combined biosynthetic pathway for de-novo production of UDP-galactose. Catalysis with multiple enzymes immobilized on agarose beads. ChemBioChem 3:348-355
Liu R, Xu Y, Chen M, Weïwer M, Zhou X, Bridges AS, Deangelis PL, Zhang Q, Linhardt RJ, Liu J (2010) Chemoenzymatic design of heparan sulfate oligosaccharides. J Biol Chem 285:34240-34249

Ludwigs U, Elgavish A, Esko JD, Meezan E, Roden L (1987) Reaction of unsaturated uronic-acid residues with mercuric-salts - cleavage of the hyaluronic-acid disaccharide 2-acetamido-2-deoxy-3-O(beta-D-gluco-4-enepyranosyluronic acid)-D-glucose. Biochem J 245:795-804

Masuko S, Higashi K, Wang Z, Bhaskar U, Hickey AM, Zhang F, Toida T, Dordick JS, Linhardt RJ (2011) Ozonolysis of the double bond of the unsaturated uronate residue in low-molecular-weight heparin and K5 heparosan. Carbohydr Res 346:1962-1966

McCormick C, Duncan G, Goutsos KT, Tufaro F (2000) The putative tumor suppressors EXT1 and EXT2 form a stable complex that accumulates in the Golgi apparatus and catalyzes the synthesis of heparan sulfate. Proc Natl Acad Sci U S A 97:668-673

Orellana A, Mohnen D (1999) Enzymatic synthesis and purification of $[3 \mathrm{H}]$ uridine diphosphate galacturonic acid for use in studying Golgi-localized transporters. Anal Biochem 272:224-231

Pandit KK, Smith JE (1993) Capsular hyaluronic-acid in Pasteurella multocida type-A and its counterpart in type-D. Res Vet Sci 54:20-24

Price KN, Tuinman A, Baker DC, Chisena C, Cysyk RL (1997) Isolation and characterization by electrospray-ionization mass spectrometry and high-performance anion-exchange chromatography of oligosaccharides derived from hyaluronic acid by hyaluronate lyase digestion: observation of some heretofore unobserved oligosaccharides that contain an odd number of units. Carbohydr Res 303:303-311

Rabenstein DL (2002) Heparin and heparan sulfate: structure and function. Nat Prod Rep 19:312-331

Rimler RB (1994) Presumptive identification of Pasteurella multocida serogroups A, D and F by capsule depolymerisation with mucopolysaccharidases. Vet Rec 134:191-192

Rusnati M, Vicenzi E, Donalisio M, Oreste P, Landolfo S, Lembo D (2009) Sulfated K5 Escherichia coli polysaccharide derivatives: a novel class of candidate antiviral microbicides. Pharmacol Ther 123:310-322

Sismey-Ragatz AE, Green DE, Otto NJ, Rejzek M, Field RA, Deangelis PL (2007) Chemoenzymatic synthesis with distinct Pasteurella heparosan synthases: monodisperse polymers and unnatural structures. J Biol Chem 282:28321-28327

Sugiura N, Shimokata S, Minamisawa T, Hirabayashi J, Kimata K, Watanabe H (2008) Sequential synthesis of chondroitin oligosaccharides by immobilized chondroitin polymerase mutants. Glycoconjugate J 25:521-530

Sugiura N, Baba Y, Kawaguchi Y, Iwatani T, Suzuki K, Kusakabe T, Yamagishi K, Kimata K, Kakuta Y, Watanabe H (2010) Glucuronyltransferase activity of $\mathrm{KfiC}$ from Escherichia coli strain K5 requires association of KfiA. J Biol Chem 285:1597-1606

Vann WF, Schmidt MA, Jann B, Jann K (1981) The structure of the capsular polysaccharide (K5 antigen) of urinary-tract-infective Escherichia coli 010:K5:H4. A polymer similar to desulfoheparin. Eur J Biochem 116:359-364

Wang Z, Ly M, Zhang F, Zhong W, Suen A, Hickey AM, Dordick JS, Linhardt RJ (2010) E. coli K5 fermentation and the preparation of heparosan, a bioengineered heparin precursor. Biotechnol Bioeng 107:964-973

Wu J-R, Chen P-Y, Shien J-H, Shyu C-L, Shieh HK, Chang F, Chang P-C (2010) Analysis of the biosynthesis genes and chemical components of the capsule of Avibacterium paragallinarum. Vet Microbiol 145:90-99

$\mathrm{Xu}$ Y, Masuko S, Takieddin M, Xu H, Liu R, Jing J, Mousa SA, Linhardt RJ, Liu J (2011) Chemoenzymatic synthesis of homogeneous ultralow molecular weight heparins. Science 334:498-501

Yip GW, Smollich M, Götte M (2006) Therapeutic value of glycosaminoglycans in cancer. Mol Cancer Ther 5:2139-2148 